\title{
Functional Analysis of an Appressorium-Specific Gene from Colletotrichum gloeosporioides
}

\author{
Tri Puji Priyatno ${ }^{1 *}$, Farah Diba Abu Bakar², Rohaiza Ahmad Redzuan², Nor Muhammad Mahadi², Abdul Munir Abdul Murad² \\ 'Indonesian Center for Agricultural Biotechnology and Genetic Resources Research and Development, Bogor, Indonesia \\ ${ }^{2}$ School of Biosciences and Biotechnology, Faculty of Science andTechnology, Universiti Kebangsaan Malaysia, Bangi, Selangor, Malaysia
}

ARTICLE INFO

Article history:

Received May 22, 2019

Received in revised form January 5, 2020

Accepted January 28, 2020

KEYWORDS:

Appressorium formation,

Colletotrichum gloeosporioides,

Colletotrichum appressoria,

spesific gene,

gene disruption,

pathogenicity

\begin{abstract}
A novel gene (CAS2) specifically expressed during appressorium formation was isolated from Colletotrichum gloeosporioides using Differential Display RTPCR. CAS2 comprises 368 deduced amino acid residues and is $50 \%$ identical to a hypothetical protein from Chaetomium globosum. ProtFun 2.2 server analysis predicted that Cas2 functions as a transport and binding protein. Based on putative transmembrane domain prediction software (HMMTOP), Cas2 protein is composed of five alpha-helical transmembrane domains with a very short external $\mathbf{N}$-terminus tail and long internal C-terminus. ExPASy ScanProsite analysis showed the presence of integrin beta chain cysteine-rich domain, $\mathrm{N}$-myristoylation site, EGF-like domain, $2 F e-2 S$ ferredoxins, iron-sulfur binding region, VWFC domain, fungal hydrophobins signature, membrane lipoprotein lipid attachment site, and Janus-faced atracotoxin (J-ACTX) family signature in CAS2 protein. Mutants with deleted $C A S 2$ were not significantly different in terms of vegetative growth, conidiation, and appressoria production compared to wild type. However, the Cas2 mutant produced multipolar germination, a feature which distinguishes it from wild type strain. Interestingly, the mutant is non-virulent to mango fruits, indicating that $C A S 2$ may encode proteins that function as novel virulence factors in fungal pathogens.
\end{abstract}

\section{Introduction}

Anthracnose disease, caused by Colletotrichum gloeosporioides or Glomerella cingulata, is very common and destructive on numerous crop and ornamental plants worldwide. This fungal pathogen is one of the best-studied species among hemibiotrophic fungi for elucidating various aspects of the host-pathogen interaction with its host. The pathogenicity of $C$. gloeosporioides depends on cellular morphogenesis event. Beginning with conidial attachment onto host surfaces, appropriate physicals and chemicals from host plant induced the conidia to germinate. Subsequently, the tip of the germ tube becomes attached to the surface and begins to swell to form a dome-shaped, highly melanized infection cell, the appressorium (Hamer et al. 1988). Next, a penetration peg emerges from a small area, adhering appressorium against the host surface (Perfect et

\footnotetext{
* Corresponding Author

E-mail Address: isdihar@yahoo.co.uk
}

al. 1999). The fungus then uses enormous turgor pressure generated in the appressorium to drive the penetration peg through underlying plant surface (Balhadère and Talbot 2001). This morphogenesis is a complex process from initiation to maturation, and involves the expression of a number of genes. Identification and characterization of genes that are active during conidial-appressorium morphogenesis is important to understand the molecular mechanisms of fungal differentiation and pathogenesis, and to develop new control methods that are rationally designed with specific targets in mind.

Several genes have been identified in $C$. gloeosporioides that are specifically expressed during appressorium formation er genes involved in the process that have not been discovered (Hwang and Kolattukudy 1995). The first appressorial genes identified, in/24 and in/26, were isolated from the rust fungus $U$. appendiculatus by differential screening of a genomic library (Xuei et al. 1992). in/24 is expressed when appressoria begin to mature and its expression is maintained throughout maturation. Likewise, 
in/26 is upregulated during appressorial maturation, although it is constitutively expressed at low levels in non-differentiated cells. The functions of these genes are unknown. Using the same approach, two appressorium-specific genes (Mi/23 and $\mathrm{Mi} / 29$ ) were identified from $M$. grisea (Lee and Dean 1993) and their functions are also unknown. An additional $M$. grisea gene, MPG1, was isolated by differential cDNA cloning and is abundantly expressed during appressorial differentiation and early plant infection (Talbot et al. 1996) during conidiation and in mycelial cultures starved for nutrient, but the importance of this gene was demonstrated by showing that mpglmutants were impaired in appressorium formation. The protein encoded by MPG1 is a hydrophobin and in addition to its role in spore and appressorium adhesion, it may also act as a developmental sensor for appressorium formation (Talbot et al. 1996).

In addition to MPG1, a PTH11 gene from $M$. grisea was predicted to encode an appressorial transmembrane protein. PTH11 was identified by REMI (Restriction Enzyme Mediated Integration) mutation (DeZwaan et al. 1999), and pth11 mutants failed to form appressoria on inductive surfaces and have decreased pathogenicity. However, these mutants were responsive to exogenous cAMP, which helps in forming functional appressoria and restoring pathogenicity. A Pth11-GFP fusion protein was found to be localized at the cell membrane. Based on these results, it was suggested the Pth11 protein plays a role in activating appressorium signaling as a receptor for inductive surface cues.

Differential display was used to isolate a novel appressorium-specific genes (CgCAS2). The sequence of the gene was used to characterize and predict the features and function of the resulting protein. A gene knockout experiment was also performed to observe the gene's function in appressoria formation and pathogenesis.

\section{Materials and Methods}

\subsection{Fungal and Culture Conditions}

C. gloeosporioides PeuB was obtained from the stock culture collection of School of Biosciences and Biotechnology, Universiti Kebangsaan Malaysia. The fungal cultures were grown by frequent subculturing on Potato Dextrose Broth (PDA: Difco, USA). Conidia, germinating conidia, appressoria, and mycelia were cultivated and harvested as described by Kamaruddin et al. (2007), (Rohaiza 2007).

\subsection{Genomic DNA and RNA Isolation}

Total DNA of C. gloeopsorioides was isolated from mycelia using the method described by Pitch and Pich and Schubert (1993). Total RNA of conidia, germinating conidia, and mycelia were extracted using TRI REAGENT ${ }^{\circledR}$ solution (Molecular Research Center, USA) while RNA from the appressoria was extracted using TRIZOL ${ }^{\circledR}$ solution in combination with mechanical cell disruption by glass beads (Rohaiza 2007) of the DNA and RNA was tested using agarose gel electrophoresis. Both DNA and RNA were stored at $-20^{\circ} \mathrm{C}$ until further usage.

\subsection{Cloning and Sequencing of $\mathrm{Cg}$ CAS2}

Isolation of genes active at the appressoria developmental stage (CgCAS2) is based on a differential display of mRNA by reverse transcription polymerase chain reaction (PCR) using arbitrary primers. A conidia suspension at $10^{6}$ conidia $/ \mathrm{ml}$ was induced and incubated for 7 hours to form appressoria on a glass Petri dish (15 cm in diameter) waxed with rubber leaves and papaya fruit wax. Total RNA from appressoria and mycelia was isolated by a modified method used by Clark (1998) trary ACP primers were used to perform independent reverse transcription PCR reactions by employing a method of GeneFishing ${ }^{\mathrm{TM}}$ (SeeGene, Korea). After separation on $2 \%$ agarose gel, the PCR products showing differential expression in appressoria (compared to mycelia) were cloned into PCR2.1-TOPO vectors using TOPO-TA Cloning Kit (Invitrogen, USA). DNA Walking Kit (SeeGene, Korea) was then used to obtain the fulllength sequence of $\mathrm{CgCAS2}$.

Three target specific primers (TSPs, Table 1) were designed from the newly-obtained CgCAS2 sequence using a DNAWalking SpeedUp ${ }^{\mathrm{TM}}$ Premix Kit (Seegene, Korea). Nested PCR was performed by using the DNA Walking Annealing Control Primers (DW-ACP) provided in the Kit and the three TSPs. Each of the DW-ACPs contained a specific ACP primer-binding site at its 3'-end (5'-AGGTC, 5'-TGGTC, 5'-GGGTC, 5 '-CGGTC). The amplification contained $100 \mathrm{ng}$ of C. gloeosporioides genomic DNA, $4 \mu \mathrm{l}$ of $2.5 \mu \mathrm{M}$ DWACP (one of DW-ACP 1, 2, 3, and 4), $1 \mu$ of $20 \mu \mathrm{M}$ TSP, $1,25 \mu$ of $2 \times$ SeeAmp $^{\mathrm{TM}}$ ACPTM Master Mix II, and sufficient distilled water to make up a $50 \mu \mathrm{l}$ reaction. In the second PCR, four PCR reactions were set up, each of which contained $3 \mu \mathrm{l}$ of the purified PCR product, $1 \mu \mathrm{l}$ of the $10 \mu \mathrm{M}$ DW-ACPN provided in the kit, $1 \mu$ lof $20 \mu \mathrm{M}$ TSP $2,10 \mu$ of $2 \times$ SeeAmp $^{\mathrm{TM}}$ 
Table 1. List of oligonucleotide primers used in this study

\begin{tabular}{lll}
\hline Name & Sequence & Sequence \\
\hline HpTsp1 & GGTGACGACAATGAT & PCR CgCAS2 ORF \\
& TTCT & \\
HpTsp2 & CCCAGTCCCACTTGT & PCR CgCAS2 ORF \\
& TGT & \\
HpTsp3 & TGTCACCCAGTTATT & PCR CgCAS2 ORF \\
& TGCT & \\
Hpw-F & CCGAGGCATAAACC & PCR CgCAS2 ORF \\
& AGGGAC-GAG & \\
Hpw-R & TGATCCCGTTGGC & PCR CgCAS2 ORF \\
& TTTGCCTTG & \\
TrpC-F & CCATGTCAACAAGA & PCR integration gene \\
& ATAAAACGC & replacement vector \\
\hline
\end{tabular}

ACPTM Master Mix II, and $5 \mu$ of distilled water to make up a $20 \mu$ reaction. In the third PCR, four PCR reactions were prepared, each of which contained $1 \mu \mathrm{l}$ of the second PCR products, $1 \mu \mathrm{l}$ of the $10 \mu \mathrm{M}$ universal primer provided in the kit, $1 \mu \mathrm{l}$ of $10 \mu \mathrm{M}$ TSP 3, $10 \mu$ l of $2 \times$ SeeAmp $^{\text {TM }}$ ACP $^{\text {TM }}$ Master Mix II, and $7 \mu \mathrm{l}$ of distilled water to make up a $20 \mu$ reaction. All of the PCRs were performed on a PCR Thermal Cycler. The extracted PCR products were cloned into the PGEM $^{\circledR}$-T Easy Vector System (Promega) and sent to a commercial DNA sequencing service (First Base, Malaysia) for nucleotide sequence determination. After the upstream CgCAS2 sequence was cloned and sequenced, two primers (Hpw-F and Hpw-R) were used to obtain the whole $\mathrm{CgCAS2}$ gene.

\subsection{Transformation-mediated Gene Replacement}

Preparation of sphaeroplasts and transformation of C. gloeosporioides were performed according to methods described by Rodriguez and Redman (1992) gromycin transformants were selected on regeneration medium containing hygromycin B $(300 \mu \mathrm{g} / \mathrm{ml})$ (Sigma, USA). Before transformation, pN1389-PKAC was linearized with Kpn1 restriction endonuclease and precipitated with ethanol. Subsequently $20 \mu \mathrm{g}$ of DNA was transfected into $C$. gloeosporioides sphaeroplasts.

\subsection{Genomic DNA and RNA Blot Analysis}

DNA digestion, agarose gel fractionation, labeling of probes and hybridization were performed according to the kit manufacturer's instruction and standard methods (Sambrook and Russel 2001) 2.5 $\mathrm{kb}$ fragment of CgPKAC DNA probe was labeled with $\left[\alpha^{-32} \mathrm{P}\right] \mathrm{dCTP}$ using Ready To $\mathrm{Go}^{\mathrm{TM}}$ DNA Labeling kit (-dCTP) (Amersham, USA). Hybridization was carried out with hybridization buffer $\left(1 \mathrm{M} \mathrm{Na}_{2} \mathrm{HPO}_{4} \cdot 2 \mathrm{H}_{2} \mathrm{O}, 1\right.$
$\mathrm{M} \mathrm{NaH}_{2} \mathrm{PO}_{4}, 0.5 \mathrm{M} \mathrm{EDTA}, 0.1 \%$ [w/v] SDS) at $65^{\circ} \mathrm{C}$ for $4 \mathrm{hrs}$ for pre-hybridization and hybridized overnight after the labeled-probes were added. The membrane was washed at $65^{\circ} \mathrm{C}$ with $2 \times$ SSC for $10 \mathrm{~min}$ followed by $2 \times$ SSC and $0.1 \%$ SDS, $1 \times$ SSC and $0.1 \%$ SDS, and $0.5 \times$ SSC and $0.1 \%$ SDS until the radioactivity signal was low. The washed blots were exposed to Fujifilm for various times at $-80^{\circ} \mathrm{C}$.

\subsection{Appressorium Induction on Hydrophobic Hard Surface}

Induction of appressorium was tested on a glass slide coated with rubber wax. A total of $50 \mu \mathrm{l}$ of wax (in chloroform) was spread on glass slide with cotton bud. Subsequently, $25 \mu \mathrm{l}$ of conidia suspension containing $10^{5}$ conidia/ml were applied on the glass slides. Appressorium formation was observed every hour for 8 hours.

\subsection{Virulence Assay}

Test for pathogenicity was performed as described by Kim et al. (2001). Mature green mangos were infected with conidia of $C$. gloeosporioides. Two modes of inoculation were applied in the pathogenicity test: inoculation on unwounded and wounded mango fruits. Before inoculation, fruits were surface sterilized with $70 \%$ ethanol and left to dry at room temperature. A total of $0.5 \mathrm{ml}$ of conidial suspensions at $2 \times 10^{4}$ conidia/ml was applied to the surface of unwounded fruits by spraying the inoculum with a spray gun (Preval, USA), while wounded fruits were inoculated with $20 \mu \mathrm{l}$ of condial suspension. Mangoes were arranged in moistened plastic trays and incubated at $30^{\circ} \mathrm{C}$ for two weeks to observe the disease symptoms. Number of lesions was observed daily.

\section{Results}

\subsection{Sequence Analysis of the CgCAS2 Gene}

A total of 2,150 bp of DNA sequence, which includes the CgCAS2 ORF, 900 bp of promoter region, and $39 \mathrm{bp}$ of 3'-end regulatory region, was obtained (Figure 1). The CgCAS2 encodes a protein with 368 amino acids. A CCCAATGTTG sequence at nucleotide position 901 to 903, complying with the Kozak's rule, was found at the start region of the ORF (Figure 1). Comparison between the sequence of the gene and its cDNA sequence revealed a 1,214 bp ORF, which is interrupted by two introns at nucleotide positions 718 to 771 and 1,003 to 1,058 , respectively. 
-900 ggcccgacgtcgcatgctcccggccgccatggcggccgcgggaattcgatttcacagaag

-840 tatgccaagcgagggggggggtctagacgatcgtgcagacatcttcaacgactggttttc

-780 aataggcgtcaatgagctgcaattggaatggtgatttgggttcgtctcgtgtcaaacaga

-720 agcttcgcctacccaagccagtgggagggaatgtgggcaaatagatagcagagagcaag

-660 ctagcaatacggtacagaatttcgggcgaccttcttgcgtctgctgtgcaatcaaacaaa

-600 gcggtttgacctcaggaaacagaaggtgtcgcccaaaatgtcactgacatgtctgtccgc

-540 agaggcacgggcttgggggaaaatgtgtcaaacccaactaccgaccgtcctgaccgaggc

-480 ataaaccagggacgagagctgcgtcaagaaaccttcgcactcgtttacagtcgcatacta

-420 tcatagatctgcgtggcatctgagcgatcgcatcgtcccgtttgggttagagcgccgtct

-360 ccagccgcgcacaacgctgaatggtcccctcatttgatgtgcagcgaaccaatgcacgga

-300 tgctgcaacttcatgctctggcacatcctcggtgagagattggccagttcgatctcgtgg

-240 cgcggtttgggaacctcggctcagcttcccggaatatggtttgcagggggttggagttgc

-180 ttctgctcctggttgaaacgtgatcgtttcgactacaagattgaagtagcccccgatgat

-120 acttggaacatcaatgggaccacgaaaatcatccgactattttcgtttctgtatactgat

-60 tcaattgtgaactacacaatggctttccaccaaaaggttttccggtcgatttattgccca

1 ATGTTGTTGCCCAATGGGGGAGAAATCATACCCAACCCGTTTCTATCAATACCCATTGCC $\begin{array}{llllllllllllllllllll}M & L & L & P & N & G & G & E & I & I & P & N & P & F & L & S & I & P & I & A\end{array}$

61 GCCGGGCTAGCAGTGGCCGCAGCGCAAGGTCACGGAGCAATTAGGATTTCCGGAGTTAAA $\begin{array}{llllllllllllllllllllllllllllllllll}A & G & L & A & V & A & A & A & Q & G & H & G & A & I & R & I & S & G & V & K\end{array}$

121 CCCGAAAATGTTCGCAGACTAGCCTACATCGCTGCGCTCGCTAGTTTTGTTCTCTCGACG $\begin{array}{llllllllllllllllllll}P & E & N & V & R & R & L & A & Y & I & A & A & L & A & S & F & V & L & S & T\end{array}$

181 ACGGAATATCTCAACAAATGGTCAGCAAATAACTGGGTGACAGACAACAAGTGGGACTGG $\begin{array}{llllllllllllllllllll}T & \mathrm{E} & \mathrm{Y} & \mathrm{L} & \mathrm{N} & \mathrm{K} & \mathrm{W} & \mathrm{S} & \mathrm{A} & \mathrm{N} & \mathrm{N} & \mathrm{W} & \mathrm{V} & \mathrm{T} & \mathrm{D} & \mathrm{N} & \mathrm{K} & \mathrm{W} & \mathrm{D} & \mathrm{W}\end{array}$

241 GATCGAGAAATCATTGTCGTCACCGGCGGCAGCAGCGGCATCGGCGCAAGCATCATCAAG $\begin{array}{llllllllllllllllllllllllllllll}D & R & E & I & I & V & V & T & G & G & S & S & G & I & G & A & S & I & I & K\end{array}$

301 CACATCTTCGCAAGAAACCCCAAAGCGACCATTGTAGTGGTTGACTTGGCACCGTTATCA $\begin{array}{lllllllllllllllllllllllllll}H & I & F & A & R & N & P & K & A & T & I & V & V & V & D & L & A & P & L & S\end{array}$

361 TGGGAACCACCCAAGGGCTCCAAGCTTCACTACTTCAAGTGTGACCTGACCGACACGGCG $\begin{array}{llllllllllllllllllll}W & E & P & P & K & G & S & K & L & H & Y & F & K & C & D & L & T & D & T & A\end{array}$

421 GCACTGAAGACGCTTTGCACTCTCATTCGAACTCAGGTTGGGGATCCTACGGTTCTCATC $\begin{array}{llllllllllllllllllll}A & L & K & T & L & C & T & L & I & R & T & Q & V & G & D & P & T & V & L & I\end{array}$

481 AATAATGCCGGCATTGCGCGGGGTGCAACAATTATGGAAGGCTCATATGCCGACATTGAG $\begin{array}{llllllllllllllllllll}N & N & A & G & I & A & R & G & A & T & I & M & E & G & S & Y & A & D & I & E\end{array}$

541 CTCACCGTGAAGACAAATCTCATTGCGCCCTTCCTGTTGACGAAGGAGTTCCTGCCGTAT $\begin{array}{llllllllllllllllllll}\mathrm{L} & \mathrm{T} & \mathrm{V} & \mathrm{K} & \mathrm{T} & \mathrm{N} & \mathrm{L} & \mathrm{I} & \mathrm{A} & \mathrm{P} & \mathrm{F} & \mathrm{L} & \mathrm{L} & \mathrm{T} & \mathrm{K} & \mathrm{E} & \mathrm{F} & \mathrm{L} & \mathrm{P} & \mathrm{Y}\end{array}$

601 ATGGTTCGCAGGAATCATGGACATATCGTCAACATCGGGTCGATGAGTTCGGTGGTCCCA $\begin{array}{lllllllllllllllllllllll}M & V & R & R & N & H & G & H & I & V & N & I & G & S & M & S & S & V & V & P\end{array}$

661 CCCGTCAGAATCGCAGATTATTCTGCAACTAAAGCAGGACTAACTGCCATGCATGAGgtc $\begin{array}{lllllllllllllllllll}P & V & R & I & A & D & Y & S & A & T & K & A & G & L & T & A & M & H & E\end{array}$

721 agtctactggtgaccacgecccaaggccgacgcttgactgacatagtacagTCTTTGCAA

781 CTCGAGTTGAAGTACATCCACAAAGCACTGAAAGTTCGACAAACGCTTGGAATCTTCGGC $\begin{array}{llllllllllllllllllll}L & E & L & K & Y & I & H & K & A & L & K & V & R & Q & T & L & G & I & F & G\end{array}$

841 TTCATCAGGACGCCTCTTGTTCCGTTCAACCCCGGACAGCCACATTTCGTTATGCCACTG $\begin{array}{lllllllllllllllllllllllllll} & \mathrm{I} & \mathrm{I} & \mathrm{R} & \mathrm{T} & \mathrm{P} & \mathrm{L} & \mathrm{V} & \mathrm{P} & \mathrm{F} & \mathrm{N} & \mathrm{P} & \mathrm{G} & \mathrm{Q} & \mathrm{P} & \mathrm{H} & \mathrm{F} & \mathrm{V} & \mathrm{M} & \mathrm{P} & \mathrm{L}\end{array}$

901 CTTCATGTCGATACTGTTGGTGAGGCAATTGTTAATGGACTTTACAGCGGATACGGCGGG $\begin{array}{lllllllllllllllllllll}\mathrm{L} & \mathrm{H} & \mathrm{V} & \mathrm{D} & \mathrm{T} & \mathrm{V} & \mathrm{G} & \mathrm{E} & \mathrm{A} & \mathrm{I} & \mathrm{V} & \mathrm{N} & \mathrm{G} & \mathrm{L} & \mathrm{Y} & \mathrm{S} & \mathrm{G} & \mathrm{Y} & \mathrm{G} & \mathrm{G}\end{array}$

961 ACCATTTACCTTCCTAGAATCATGTCTTTGGTGACTGCACTCgtaagttgtaaattatc $\begin{array}{lllllllllllllll}T & I & Y & L & P & R & I & M & S & L & V & T & A & I\end{array}$

1021 ccaaaacaattagcatggggctaacagaatcaaacagAGGGCAGGGCCGGAATGGATA

$$
\begin{array}{lllllll}
R & A & G & P & E & W & I
\end{array}
$$

1081 TGGCGCCTAGCGCGAGAGACAACCGCCAGTGCAAAGGATATCCCTTACACCCCCCGCCAG $\begin{array}{llllllllllllllllllllll}W & R & L & A & R & E & T & T & A & S & A & K & D & I & P & Y & T & P & R & Q\end{array}$

1141 AAGATTAATGACTTGACGGGCACGTTTGACTTGGAAGAGGCTAGCAAGGCAAAGACCAAC $\begin{array}{llllllllllllllllllll}K & I & N & D & L & T & G & T & F & D & L & E & E & A & S & K & A & K & T & N\end{array}$

1201 GGGATCAAGAATGAGCTTTAATCATCGAAACATTTCATCAATGTAAATAAGTATATTTTG G I $\quad \mathrm{K} \quad \mathrm{N} \quad \mathrm{E} \quad \mathrm{L} \quad-$

Figure 1. Nucleotide sequence of CgCAS2 fragment showing the deduced amino acid residues and the two intron regions within the ORF. The deduced amino acids (368 residues) are indicated with abbreviations and shown below the ORF. Intron sequences are shown in lower case red letters and underlined. The potential CAAT box, TATA box, GAGA factor, factor NF-E1/NF-E1a/NF-E1b/NF-E1c binding site (YTATCW), transcription factor NF-Y/CTF/CBF binding sites (ATTGG) and polyadenylation (ATAATAA) are underlined and marked in blue letters 
Analysis of the $900 \mathrm{bp}$ upstream sequence of the coding region indicates that the 5' flanking region of the CgCAS2 contains several potential regulatory elements (Figure 1). TATA box is absent in the CgCAS2 promoter. However, a TATA-like sequence was detected at position -66 bp upstream of ATG. RNA polymerase II CCAAT signal was identified at $-311 \mathrm{bp}$ upstream of the start codon. Other putative regulatory elements identified at the upstream sequence are the GAGA factor binding site at $-266,-466$, and -668 , factor NF-E1/NF-E1a/NF-E1b/NF-E1c binding site (YTATCW) at -422 and transcription factor NF-Y/CTF/ CBF binding sites (ATTGG) at -261 and -758 . Within the 3'-untranslated region, a putative polyadenylation sequence (5'-AAATAA-3') is detected at the position 1,244-1,249 downstream from the ATG (Figure 1).

The predicted $\mathrm{CgCAS2}$ protein has a theoretical molecular mass of $41.7 \mathrm{kDa}$ and a calculated isoelectric point of 9.4. PSORT (http://psort.nibb. ac.jp) analysis showed that there is a $65.2 \%$ possibility that this protein is located in the cytoplasm, $17.4 \%$ in the mitochondria, $13 \%$ in the nucleus and $4.3 \%$ in the endoplasmic reticulum. Analysis of the $\mathrm{N}$-terminal amino acid sequence using SignalP software predicted the presence of a signal sequence that is 24 amino acids long. Similarity search against known proteins showed that the deduced amino acid sequence of $\mathrm{CgCAS2}$ shares significant homology with some hypothetical proteins from other fungi, and the highest hits were with a hypothetical protein from C. globosum (CHGG09887) with 50\% identity, hypothetical protein from A. niger (An14g01270) with $46 \%$ identity, and hypothetical protein from $M$. grisea (MGG01604) with 40\% identity. CgCAS2 is rich in Ala (9.8\%) and Leu (10.1\%).

\subsection{Disruption of $\mathrm{CgCAS2}$}

Gene disruption was performed to test for the possible involvement of CgCAS2 in appressorium morphogenesis. To construct a gene replacement vector, a $2.3 \mathrm{~kb}$ hygromycin resistance $(h p h)$ gene cassette was inserted into HindIII site of a cloned $1.8 \mathrm{~kb}$ CgCAS2 fragment in pGEMCAS2 to generate the final construct, pGEMCAS2-hph (Figure 2a). Linear and circular versions of pGEMCAS2-hph were transfected into the sphaeroplasts of $C$. gloeosporioides wild-type strain PeuB. Schematics of the homologous integration is shown in Figure 2b.

A total of 35 hygromycin-resistant transformants were isolated by single spore isolation and subcultured on PDA plate containing $300 \mathrm{~g} / \mathrm{ml}$ hygromycin. All transformants were screened using PCR with HpF-F and HpF-R primers, which are complementary to the native CgCAS2 DNA fragment, as well as with TrpC-F and HpF-R primers. TrpC-F primer was designed based on $\operatorname{TrpC}$ terminator sequence in the hygromycin resistance gene cassette. In two transformants, Cgcas2- $x 2$ and Cgcas2-c1, HpF-F and HpF-R primers did not produce the expected $\sim 1.7 \mathrm{~kb}$ PCR fragment, indicating that there is an insertion of $h p h$ DNA fragment into the CgCAS2 locus. TrpC-F and HpF-R primers amplified a $\sim 1.5 \mathrm{~kb}$ amplicon in Cgcas2- $x 2$ and Cgcas2-c1, but not in the wild-type strain that do not have $h p h$ gene cassette insertion (Figure 2b).

Cloning and sequencing of that fragmentconfirmed that homologous integration at the CgCAS2 locus took place in the Cgcas2-c1 mutant only. The disruption of CgCAS2 in Cgcas2-c1 mutant was also confirmed by Southern blot analysis (Figure 2c, d, e). In Cgcas2-c1, three extra bands with the size of $\sim 1 \mathrm{~kb}, \sim 6 \mathrm{~kb}$, and $\sim 7$ kb were observed (Figure $2 \mathrm{c}$ ). Hybridisation with the hygromycin phosphotransferase $(h p h)$ gene showed that Cgcas2-c1 produced bands with different sizes, whereas no signals were observed for the wild type (Figure 2d). To further clarify if gene replacement had occurred within CgCAS2 locus, the genomic DNAs were digested with KpnI, which has no restriction sites in wild type CgCAS2. When it was hybridised with the $1.7 \mathrm{~kb}$ fragment of CgCAS2, only Cgcas2-c1 had a $\sim 6 \mathrm{~kb}$ fragment, in contrast to the $\sim 2.7 \mathrm{~kb}$ fragments seen in the wild-type strain (Figure 2e).

To test the expression of the CgCAS2 gene by the mutant, total RNA extracted from appressoria of the wild type and Cgcas2-c1 mutant were subjected to Northern blot analysis using the CgCAS2 cDNA as a probe. The results confirmed the absence of CgCAS2 transcripts in the appressoria of Cgcas2-c1 mutants, whereas a CgCAS2 transcript was detected in the wild-type (Figure 3).

\subsection{The Effect of CgCAS2 Disruption on C. gloeosporioides Morphogenesis}

The Cgcas2 mutant strains had the typical grayish color and colony morphology similar to the wildtype strain when grown on PDA. The growth rate of Cgcas2 mutants, which was measured on PDA Petri dish cultures, is the same as that of the wild-type after incubation at ambient temperature for one week. 
$\mathrm{a}$

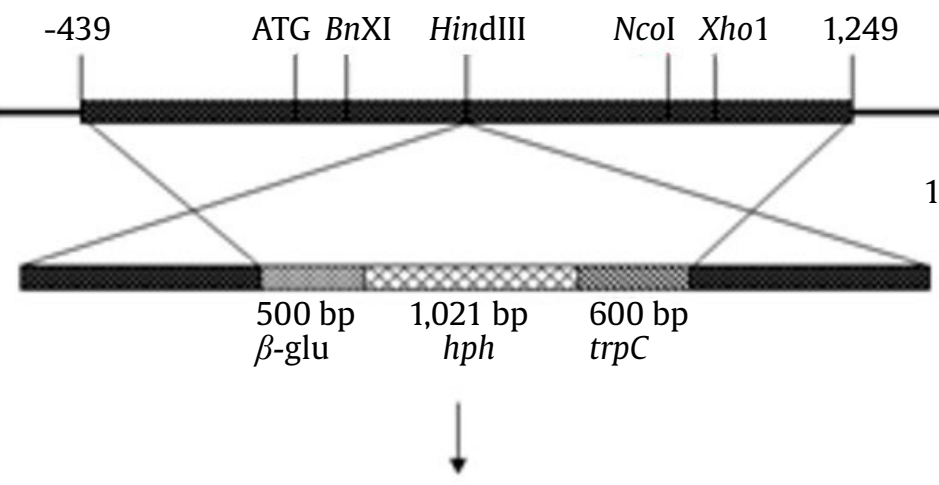

b

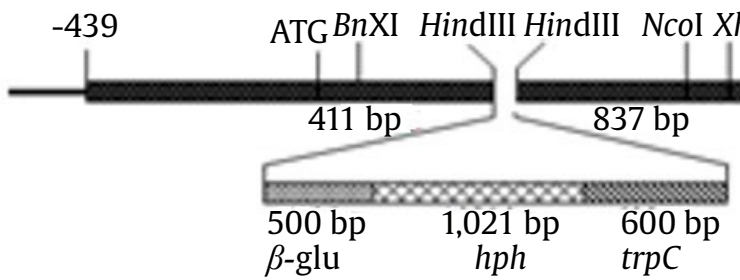

d
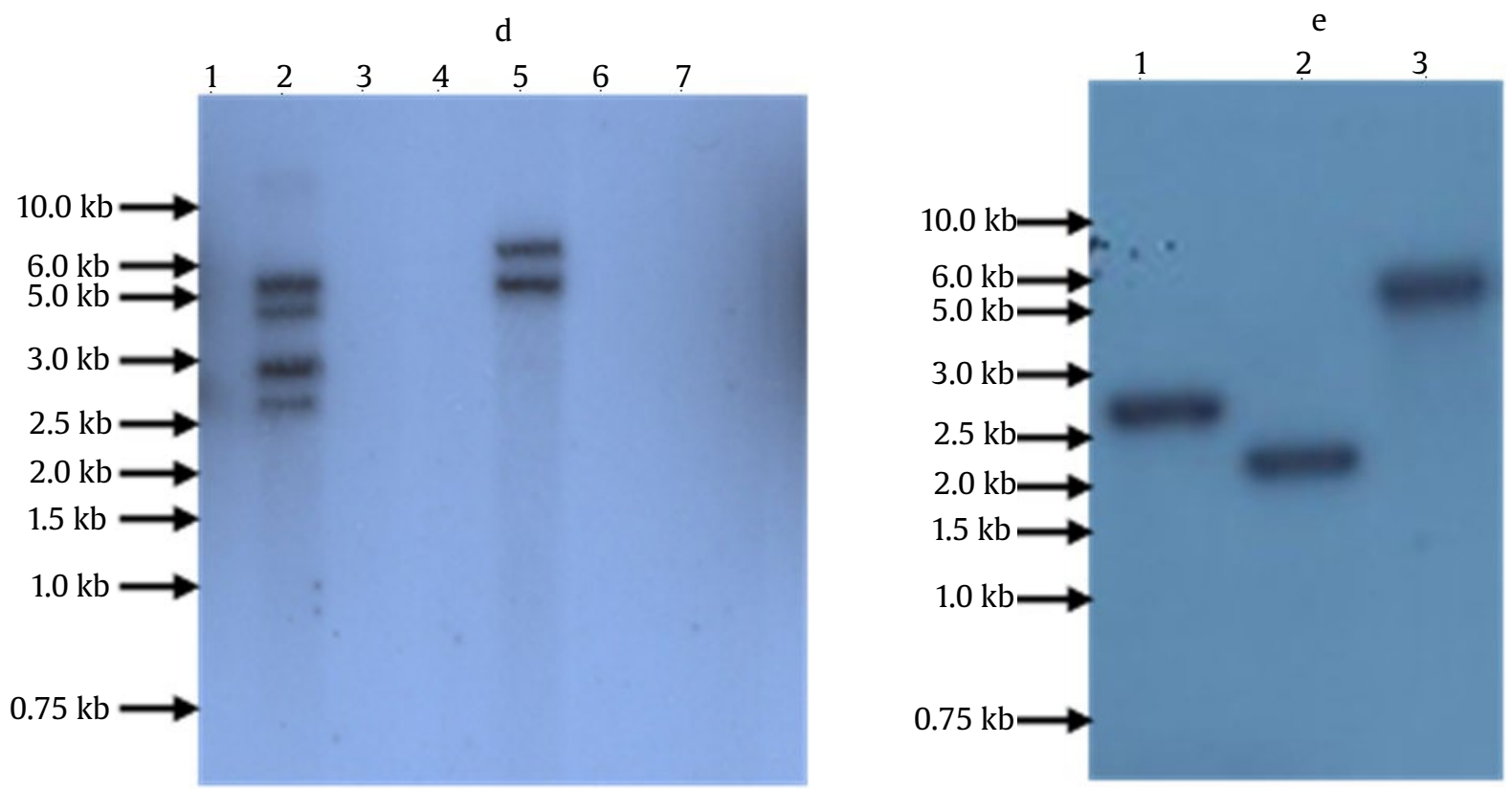

Figure 2. Schematic presentation of the strategy used for CgCAS2 gene disruption in C. gloeosporioides. (a) restriction map of the CgCAS2 locus, (b) partial map of the pGEMCAS2-hph replacement vector. DNA blot analysis of CgCAS2 gene replacement in transformant Cgcas2-x1 (lane 1), Cgcas2-x2 (lane 2), Cgcas2-x3 (lane 3), Cgcas2-x15 (lane 4), Cgcas2-c1 (lane 5), Cgcas2-c2 (lane 6), and C. gloeosporioides wild type strain PeuB (lane 7). Genomic DNA was digested with Xho1 and probed with $1.8 \mathrm{~kb}$ of CgCAS2, (c) $1.1 \mathrm{~kb}$ of $h p h$ fragments, (d) the band in Cgcas2$x 2$ and Cgcas2-c1 samples showed different patterns of DNA fragments compared to the wild type strain when hybridised with $1.8 \mathrm{~kb}$ of CgCAS2 probe. Probing with $1.1 \mathrm{~kb}$ of $h p h$ confirmed that both mutants carried the hygromcin resistant gene cassette in the mutant genome. To confirm that targeted integration has taken place in Cgcas2-x2 and Cgcas2-c1, genomic DNA was digested with KpnI and probed with $1.1 \mathrm{~kb}$ of CgCAS2 gene, (e) a single band was detected when genomic DNA was digested with KpnI and probed with $1.8 \mathrm{~kb}$ of CgCAS2 gene. In the Cgcas2-c1 mutant, an increase in $2.3 \mathrm{~kb}$ (hygromycin cassette) was observed when compared to the wild type. Lane 1: wild type; lane2: Cgcas2-x2; lane3: Cgcas2-c1 
The Cgcas2 mutant produced vegetative hyphae and abundant aerial mycelia. No obvious differences in conidial morphology was observed between the wild-type and the Cgcas2 mutant, but the amount of conidia production was slightly different (data not shown). This indicates that CgCAS2 is essential for conidiation in $C$. gloeosporioides.

The effects of CgCAS2 deletion mutant on germination and appressorium formation were assayed on hard surface glass slide coated with rubber leaf wax. Conidia produced by Cgcas2 mutants were able to germinate and form appressoria. These mutant appressoria were melanised properly and had regular shapes. However, the percentage of germ

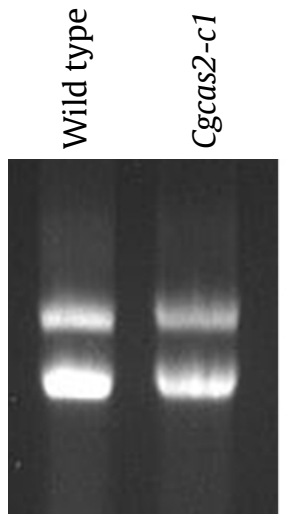

a

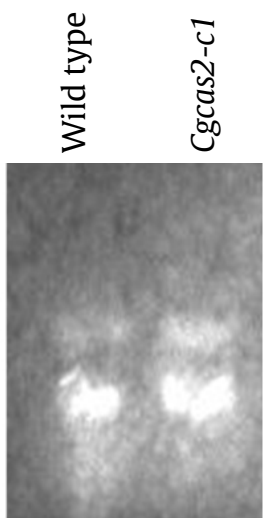

b

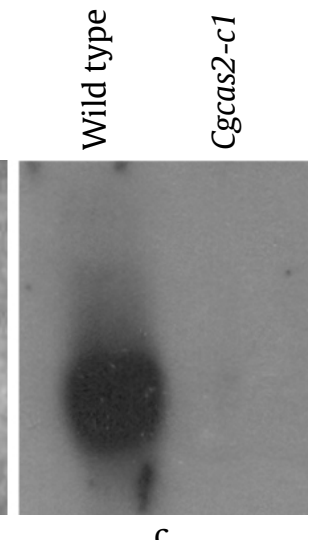

c
Figure 3. RNA blot analysis of total RNA obtained from appressoria of the wild type and the Cgcas2 mutant of $C$. gloeosporioides. (a) the total RNA was extracted from 7-hour old appressoria induced with rubber leave wax on Petri dish. RNA was electrophoresed, (b) blotted onto nitrocellulose membrane, and (c) hybridised with a $\alpha^{-32} \mathrm{P}-\mathrm{dCTP}$ labeled $1.7 \mathrm{~kb}$ fragment of CgCAS2 gene tubes and appressoria formation was significantly reduced in the Cgcas2 mutants compared to the wild-type strain (Figure 4). In addition, the Cgcas2 mutant conidia produced multipolar germination, in contrast with unipolar germination found in wild type conidia. However, appressoria differentiation only occurred at the tip of one of the germ tubes in both mutant and wild type. The remaining germ tubes in the mutant were unable to differentiate to form appressoria. The Cgcas2 mutant also produced longer germ tubes before forming appressoria, while the wild type conidia produced sessile appressoria (Figure 5).

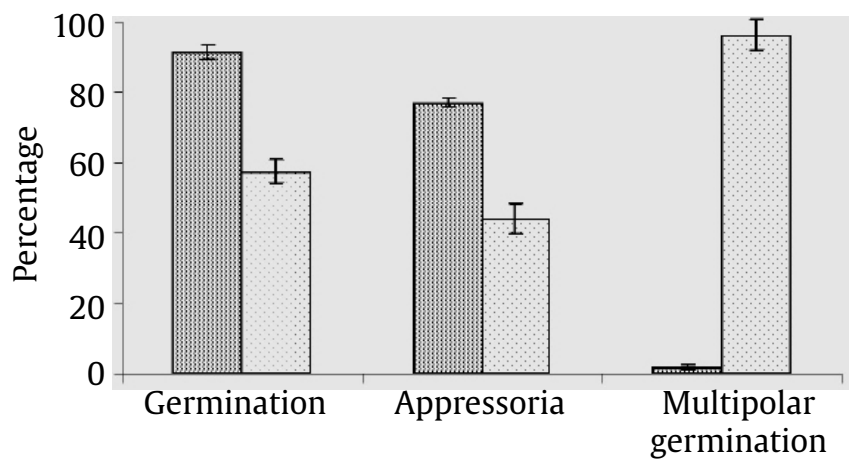

Figure 4. Percentage of germination, appressorium formation and multipolar germination of the

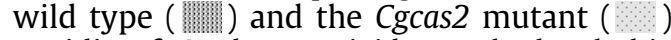
conidia of $C$. gloeosporioides on hydrophobic hard surface glass slide coated with rubber leaf wax. The mean values of the same coloured bars inscribed with a common letter are not significantly different base on statistical analysis $(\mathrm{p}<0.01)$ a

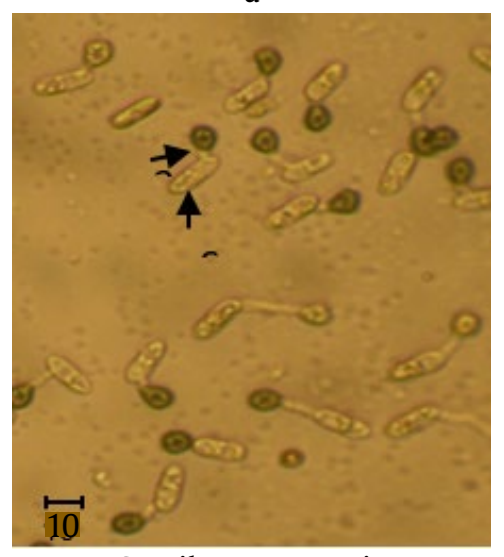

Sessile appressoria b

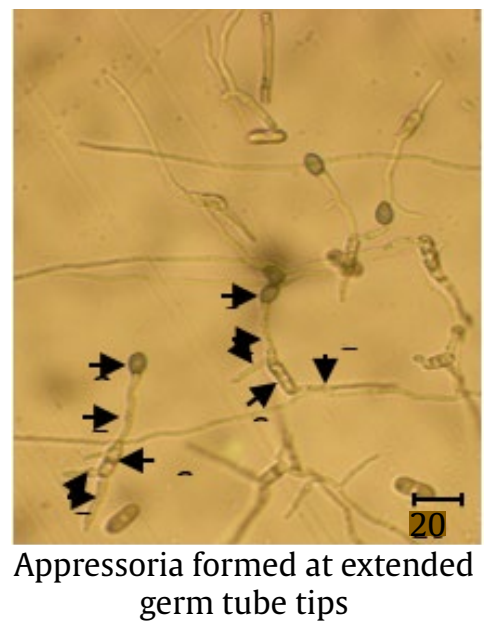
germ tube tips
C

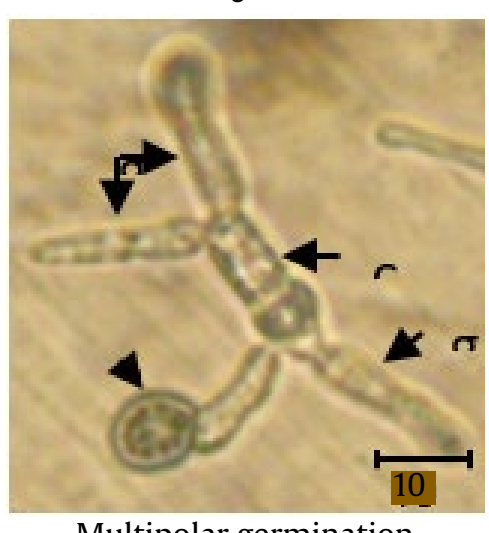

Multipolar germination

Figure 5. Light microscope observation of sessile appressorium formation in the wild type (a) and appressorium formation at extended germ tube tips of Cgcas2 mutant (b) of $C$. gloeosporioides. Multipolar germination (b, c) of Cgcas2 mutant on the hard surface of hydrophobic glass slides coated with rubber leave wax. The image was captured with an Olympus phase contrast microscope (200× magnification for a and b; 400× magnification for c) and a Nikon digital camera. (a: appressorium; c: conidium; g: germ tube) 


\subsection{CgCAS2 is Required for C. gloeosporioides Pathogenicity}

To determine the role of CgCAS2 in pathogenesis, conidia of Cgcas2 deletion mutants were inoculated onto mango fruits. Two methods of inoculation, i.e., direct inoculation onto wounded fruits and spray inoculation onto unwounded fruits, were employed to test for pathogenesis. In wounded fruits, the wild-type strain induced typical brown lesions on fruits 3 days after inoculation and developed typical necrotic, sunken anthracnose symptoms 7 days after inoculation. In contrast, small brownish lesions were observed 3 days after inoculation with the Cgcas2 mutant, which did not develop into typical anthracnose symptoms seen in the wild type. Anthracnose disease severity was measured by lesion diameters and the Cgcas2 mutant induced significantly smaller lesions than the wild type strain (Figure 6 and 7). When conidia were inoculated on unwounded fruits, initial symptoms by the wild type

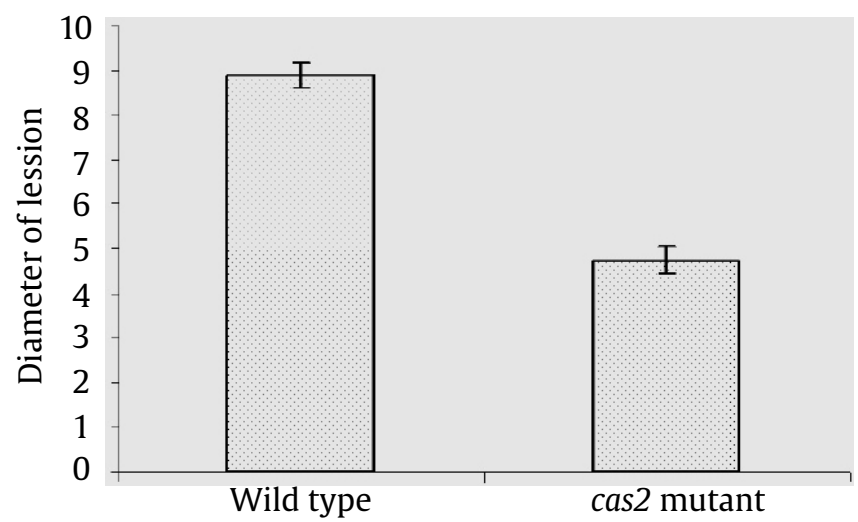

Figure 6. Disease severity of mango inoculated with the wild type and the Cgcas2 mutant of $C$. gloeosporioides

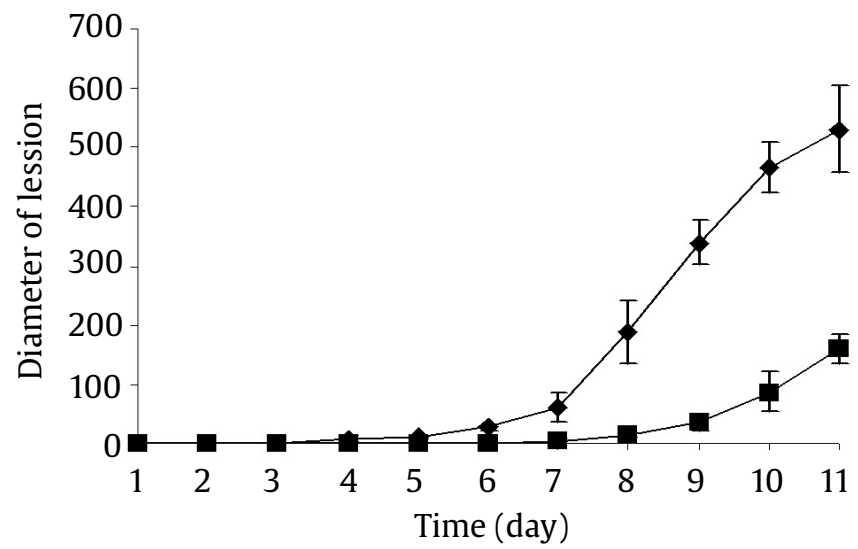

Figure 7. Disease severity of mango inoculated with the wild type $(\downarrow)$ and the Cgcas2 mutant $(\mathbf{\square}) C$. gloeosporioides strain appeared 4 days after inoculation and severe sunken lesion symptoms were observed 9 and 10 days after inoculation. However, smaller brown lesions were observed on unwounded fruits sprayed with Cgcas 2 mutant conidia 6 days after inoculation. Disease severity (based on the number of lesions) was nearly 3-fold lower in Cgcas2 mutant compared to the wild type strain. In addition, lesions induced by Cgcas 2 mutant did not further develop into typical anthracnose symptoms. These results indicate that CAS2 has an important role in pathogenesis of $C$. gloeosporioides.

\section{Discussion}

A total of 2,150 bp of CgCAS2 DNA sequence consist of 1,214 bp of ORF, $900 \mathrm{bp}$ of promoter region, and $39 \mathrm{bp}$ of 3'-end regulatory region. Confirmation of the ORF sequence and its CDNA sequence revealed that a 1,214 bp ORF was interrupted by two introns at nucleotide positions 718 to 771 and 1,003 to 1,058 , respectively. The intron/exon splice junction (GTA[Y/A] $\mathrm{GT} /[\mathrm{A} / \mathrm{C}] \mathrm{AG}$ ) of the two introns are typical of splice site sequences in other $C$. gloeosporioides genes and fit the consensus sequences found in other filamentous fungi. The second intron has the internal splicing sequence GCTAACPr necessary for lariat formation in filamentous fungi (Ballance 2017).

The 900 bp of 5' upstream sequence of the ORF CgCAS2 contains several potential regulatory elements without TATA box. Genes from filamentous fungi often lack classical regulatory sequence of the 5' and 3' non-coding regions of other eukaryotes, and some filamentous fungi promoters do not contain any TATA boxes (Ballance 2017). However, a TATA-like sequence was detected at position -66 bp upstream of ATG. Within the 3'-untranslated region, a putative polyadenylation sequence (5'-AAATAA-3') motif is required for proper RNA cleavage and subsequent polyadenylation. The spacing between the CgCAS2 AAATAA element at position 1,245 and poly (A) tail is $19 \mathrm{bp}$ in length, indicating that this element is most likely recognized during RNA processing (Wolfgang et al. 1997).

ORF CgCAS2 encoding a protein with 368 amino acids is present as a single copy gene in $C$. gloeosporioides genome and uniquely expressed in the appressoria (Rohaiza 2007). A comparative analysis of CgCAS2 with known proteins from other organisms showed sequence identity to hypothetical proteins of several fungal species including $C$. globosum, A. niger and $M$. grisea. C. gloeosporioides CgCAS2 sequence contains putative casein kinase II phosphorylation site, 
glycosaminoglycan attachment site, protein kinase C phosphorylation site and short-chain dehydrogenases/ reductases. The presence of the putative kinase dependent phosphorylation motifs in CgCAS2 and the importance of kinase signaling in $C$. gloeosporioides disease pathway signify a possible role of this protein in plant infection process (Gupta and Chattoo 2007).

Interestingly, Cgcas2 deletion mutant produced multipolar germination, however, appressorium differentiation was observed only in one germ tube (the first germ tube), while the other germ tubes were unable to form appressoria. In the entomopathogenic fungus, Beauveria bassiana, Talaei-Hassanloui and Co-Worker et al. (2007) reported that conidia from non-virulent isolates germinate in multipolarity/ multidirectional in Sabouraud Dextrose Agar but not for virulent isolates. C. gloeosporioides conidia that produced bidirectional germination in rich medium and in the absence of plant signal, do not form appressoria and are reduced in virulence (Barhoom and Sharon 2004). In this study, Cgcas2 mutant was also significantly reduced in virulence and did not produce typical anthracnose symptoms but small brown lesions that are low in abundance. A simple hypothesis can be proposed whereby conidia consume more energy for producing multidirectional germination than unipolar germination, thus multipolarity germination of conidium could lower the mechanical force and reduce available enzyme processes for penetration. Therefore, it can be hypothesized that $C g C A S 2$ plays an important role in the coordination of cellular processes required for pathogenic and saprophytic development in C. gloeosporioides appressoria.

\section{Acknowledgements}

The authors are grateful to The Indonesian government and Integrated Pest Management for Smallholder Estate Crops Project-Research Component (ADB No.1469-INO SF) for financial support. The Malaysian government and the Universiti Kebangsaan Malaysia for their financial support through the IRPA Grant No: 02-01-02-SF0141, 09-02-02-0040-EA136 and 09-02-02-0105-EA257.

\section{References}

Ballance DJ. 2017. Transformation systems for filamentous fungi and an overview of fungal gene structure. In: Leong SA and Berka RM (Eds.). Molecular industrial mycology: Systems and applications for filamentous fungi. New York: Marcel Dekker. pp. 1-29.
Balhadère PV, Talbot NJ. 2001. PGE1 encodes a P-type ATPase involved in appressorium-mediated plant infection by the rice blast fungus Magnaporthe grisea. Plant Cell 13:1987-2004.

Barhoom S, Sharon A. 2004. cAMP regulation of 'pathogenic' and 'saprophytic' fungal spore germination. Fungal Genetics and Biology 41:317-326. DOI:10.1016/j. fgb.2003.11.011

Clark S. 1998. Gene expression in Glomerella cingulata during appressorium formation [Thesis]. Dunedin, Selandia Baru: University of Otago.

DeZwaan TM et al. 1999. Magnaporthe grisea Pth11p is a novel plasma membrane protein that mediates appressorium differentiation in response to inductive substrate cues. Plant Cell 11:2013-2030.

Gupta A, Chattoo BB. 2007. A novel gene MGA1 is required for appressorium formation in Magnaporthe grisea. Fungal Genetics and Biology 44:1157-1169. DOI:10.1016/j.fgb.2007.02.014

Hamer JE et al. 1988. A mechanisms for surface attachment in spores of a plant pathogenic fungus. Science 239:288290.

Hwang CH, Kolattukudy PE. 1995. Isolation and characterization of genes expressed uniquely during appressorium formation by Colletotrichum gloeosporioides conidia induced by the host surface wax. Molecular Genetics and Genomics 247:282-294.

$\mathrm{Kim} \mathrm{Y}$ et al. 2001. Inhibition of fungal appressorium formation by pepper (Capsicum annuum) esterase. Molecular Plant-Microbe Interaction Journal 14:80-85. DOI:10.1094/MPMI.2001.14.1.80

Lee YH, Dean RA. 1993. cAMP regulates infection structure formation in the plant pathogenic fungus Magnaporthe grisea. Plant Cell 5:693-700.

Perfect SE et al. 1999. Colletotrichum: a model genus for studies on pathology and fungal-plant interactions. Fungal Genetic Biology 27:186-198.

Pich U, Schubert I. 1993. Midiprep method for isolation of DNA from plants with a high content of polyphenolics. Nucleic Acids Research 21:3328-3330.

Rohaiza RA. 2007. Pengenalpastian gen yang terlibat dalam pembentukan appressorium Colletotrichum gloeosporioides dan pencirian CAS1, gen khusus appressorium. Bangi, Universiti Kebangsaan Malayssia.

Rodriguez RJ, Redman RS. 1992. Molecular transformation and genome analysis of Colletotrichum. In: Bailey JA and Jeger MJ (Eds.). Colletotrichum, biology, pathology and control. Wallingford: CABI. pp. 47-66.

Sambrook J, Russel D. 2001. Molecular Cloning: A Laboratory Manual. 3rd ed. New York: Cold Spring Harb Lab Press Cold Spring Harb.

Talbot N et al. 1996. MPG1 encodes a fungal hydrophobin involved in surface interactions during infectionrelated development of Magnaporthe grisea. Plant Cell 8:985-999.

Talaei-hassanloui $\mathrm{R}$ et al. 2007. Germination polarity of Beauveria bassiana conidia and its possible correlation with virulence. J Inverteb Pathol 94:102-107.

Wolfgang W et al. 1997. Identification of pirin, a novel highly conserved nuclear protein. J Biol Chem 272:84828489. DOI:10.1074/jbc.272.13.8482

Xuei X et al. 1992. Characterization of INF56, a gene expressed during infection structure development of Uromyces appendiculatus. Gene 110:49-55. DOI:10.1016/03781119(92)90443-S 